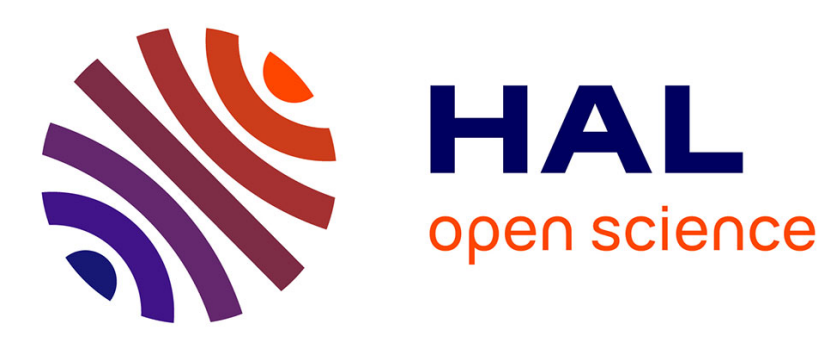

\title{
Position, Velocity, Attitude and Accelerometer-Bias Estimation from IMU and Bearing Measurements
}

\author{
Simone de Marco, Minh-Duc Hua, Tarek Hamel, Claude Samson
}

\section{To cite this version:}

Simone de Marco, Minh-Duc Hua, Tarek Hamel, Claude Samson. Position, Velocity, Attitude and Accelerometer-Bias Estimation from IMU and Bearing Measurements. ECC 2020 - European Control Conference, Mar 2020, Saint Petersburg, Russia. 10.23919/ECC51009.2020.9143918 . hal-03052517

\section{HAL Id: hal-03052517 https://hal.science/hal-03052517}

Submitted on 10 Dec 2020

HAL is a multi-disciplinary open access archive for the deposit and dissemination of scientific research documents, whether they are published or not. The documents may come from teaching and research institutions in France or abroad, or from public or private research centers.
L'archive ouverte pluridisciplinaire $\mathbf{H A L}$, est destinée au dépôt et à la diffusion de documents scientifiques de niveau recherche, publiés ou non, émanant des établissements d'enseignement et de recherche français ou étrangers, des laboratoires publics ou privés. 


\title{
Position, Velocity, Attitude and Accelerometer-Bias Estimation from IMU and Bearing Measurements
}

\author{
S. de Marco, M-D. Hua, T. Hamel and C. Samson
}

\begin{abstract}
This paper considers the problem of estimating the position, attitude and velocity of a rigid-body in a 3D space by fusing bearing measurements provided by a monocular camera with gyroscopic and accelerometer measurements provided by an Inertial Measurement Unit (IMU). The proposed deterministic observer is accompanied with an observability analysis, that points out the minimum number of image points (bearings) along with their configuration in the inertial frame, under which local exponential stability is guaranteed. The performance of the observer is demonstrated by performing experiments on a test-bed inertial-Visual sensor.
\end{abstract}

\section{INTRODUCTION}

The problem of full pose (position and attitude) estimation of a rigid body plays a major role for autonomous navigation of robotic vehicles. The orientation of a rigid body can be estimated from the output of low cost Inertial Measurement Unit (IMU) [18], whereas translational velocity and position can not be estimated from accelerometer measurement via sole integration due to unbounded growth in the estimation error caused by the sensor noise and bias [22].

In order to circumvent this problem, IMUs are usually complemented by other sensors such as Global Positioning System (GPS) which allow to bound the error [10]. However, in many applications of interest such as indoors and urban canyon environments the GPS signal may be unreliable or heavily degraded. For this reason, the usage of other type of sensors such as laser scanners (LIDAR), UltraWide Band sensors (UWB), monocular cameras and stereo cameras have been considered. Early approaches for state estimation were based on extended Kalman filters (EKF) [4], unscented Kalman filters (UKF) [16] and particle filters [1]. The last fifteen years have witnessed an increased interest in deterministic non-linear observer design for mechanical systems with symmetry that can provide stronger stability results with large domain of attraction [2], [8], [19]. Based on the type of sensors considered for the particular application at hand, several research directions have been investigated.

For instance, in the particular case of landmarks measurements (which can be retrieved by using depth cameras, range measurements and bearings, stereo cameras) an invariant extended Kalman filter (IEKF) on the group of double homogeneous matrices $\mathrm{SE}_{2}(3)$ has been proposed in [6]. In the same context, an observer on the Lie group of rigid body

S. de Marco, M-D. Hua are with I3S, Université de Nice, SophiaAntipolis, France sdemarco (hua) di3s.unice.fr.

T. Hamel is with I3S, Université Côte d'Azur, Institut Universitaire de France, CNRS, Sophia Antipolis, France, thamelei3s.unice.fr

C. Samson is with INRIA and I3S, UCA-CNRS, Sophia Antipolis, France, claude.samsondinria.fr motions SE(3) based on the Lagrange-d'Alembert principle has been derived in [15]. In case of velocity and landmark measurements a non-linear observer on $\mathrm{SE}(3)$ has been presented in [21]. Recently, a non-linear observer that estimates the position, velocity, attitude and gyro bias by fusing multiple measurements such as full position in the inertial frame (GPS), range (UWB), bearings in the inertial frame (MOCAP) and altimeter along with IMU measurements has been considered in [7].

In case of bearing measurements in the body frame, the estimation problem is referred to as Perspective-n-Point problem $(\mathrm{PnP})$. Classical methods for the solution of the PnP problem are based on algebraic approaches [11], [9] and iterative algorithms based on gradient search [13], [17]. The PnP problem has been subsequently studied in the context of observer design. In particular, in [14] an UKF like filter is derived considering only one source point and IMU measurements. The convergence of this observer depends on the motion of the system, since an implicit triangulation is required. In [3], the authors present a minimum-energy state estimator with constraints considering bearing-only measurements. A non-linear observer evolving on $\mathrm{SE}(3)$ from bearing, angular and linear velocity measurements is derived in [5]. In a prior work [12], a Riccati observer for the PnP problem based on angular and linear velocities measurements has been proposed.

In this paper, we propose a deterministic Riccati observer, based on the general framework presented in [12], providing pose, linear velocity and accelerometer-bias estimation by fusing IMU and bearings measurements, without relying on linear velocity measurements. The present work is motivated by a large number of applications in which linear velocity measurements are not available, and a reliable estimate of the linear velocity and of the pose is needed for control purposes. This is the case of low cost small-scale vehicles that operates in GPS denied environment for which the cost of body velocity sensors such as sensors based on Doppler effect or ad-hoc inertial velocity sensor systems are usually prohibitive.

\section{PRELIMINARY MATERIAL}

\section{A. Notation}

We denote by $\mathbb{R}^{n}$ the $n$-dimensional Euclidean space and by $|x|$ the Euclidean norm of the vector $x \in \mathbb{R}^{n}$. Let $\mathcal{B}_{r}^{n}:=\left\{x \in \mathbb{R}^{n}:|x| \leq r\right\}$ denotes the closed ball in $\mathbb{R}^{n}$ with radius $r$. The null matrix and the identity matrix of dimension $n \times n$ are denoted respectively by $0_{n}$ and $I_{n}$. The null matrix of dimension $m \times n$ is denoted by $0_{m \times n}$. 
The Special Orthogonal group of order three is denoted by $\mathrm{SO}(3):=\left\{R \in \mathbb{R}^{3 \times 3}: \operatorname{det}(R)=1, R^{\top} R=R R^{\top}=I_{3}\right\}$. For any $x, y \in \mathbb{R}^{3}$, we denote by $x_{\times}$the skew symmetric matrix associated to the cross product i.e. $x_{\times} y=x \times y$, where $\times$ is the vector cross product in $R^{3}$. Let $\mathbb{S}^{n}:=\{x \in$ $\left.\mathbb{R}^{n+1}:|x|=1\right\}$ denotes the $n$-dimensional sphere embedded in $\mathbb{R}^{n+1}$ with radius equal to one. For any $p \in \mathbb{S}^{2}$, we denote by $\Pi_{p}:=\left(I-p p^{\top}\right)$ the projection onto the tangent space of the unit sphere $\mathbb{S}^{2}$ at point $p$.

\section{B. Riccati Observer for a Special class of Systems}

The observer proposed in this paper is based on the Riccati Observer framework developed in [12]. In particular we consider the following class of nonlinear systems whose state $x:=\left[\tilde{x}_{1}^{\top}, x_{2}^{\top}\right]$, with $\tilde{x}_{1} \in \mathcal{B}_{r}^{n_{1}}$ and $x_{2} \in \mathbb{R}^{n_{2}}$, and outputs $y \in \mathbb{R}^{m}$ evolve according to

$$
\left\{\begin{array}{l}
\dot{x}=A\left(\tilde{x}_{1}, t\right) x+\left[\begin{array}{l}
u_{1} \\
u_{2}
\end{array}\right]+O\left(\left|\tilde{x}_{1}\right|^{2}\right)+O\left(\left|\tilde{x}_{1}\right|\left|u_{1}\right|\right) \\
y=C_{1}\left(\tilde{x}_{1}, x_{2}, t\right) \tilde{x}_{1}+C_{2}\left(\tilde{x}_{1}, x_{2}, t\right) x_{2}
\end{array}\right.
$$

where $A\left(\tilde{x}_{1}, t\right)$ is a continuous matrix-valued function uniformly bounded w.r.t. $t$ and uniformly continuous w.r.t. $\tilde{x}_{1}$ of the form

$$
A\left(\tilde{x}_{1}, t\right)=\left[\begin{array}{cc}
A_{11}(t) & 0_{n_{1} \times n_{2}} \\
A_{21}\left(\tilde{x}_{1}, t\right) & A_{22}(t)
\end{array}\right]
$$

and $C:=\left[C_{1}, C_{2}\right] \in \mathbb{R}^{m \times\left(n_{1}+n_{2}\right)}$ is a continuous matrixvalued function uniformly bounded w.r.t. $t$ and uniformly continuous w.r.t. $x$.

In the sequel $\tilde{x}_{1}$ will represent the attitude error between the camera orientation and the estimated one. We recall here that for a Riccati observer design one has to use a minimal parametrization of $\mathrm{SO}(3)$, however there are many ways to minimally parameterize $\mathrm{SO}(3)$ (e.g. Euler angles, vector part of Rodriques unit quaternion, Cardan angles). Consequently, system (1) can be seen as a handy way to take into account all the possible parameterizations, with the related nonlinear errors and first-order approximations, without choosing a priori one of them.

Let $\hat{x}_{2}$ be an estimate of $x_{2}$ and consider the following observer

$$
\left\{\begin{array}{l}
u_{1}=-K_{1}\left(y-C_{2} \hat{x}_{2}\right) \\
\dot{\hat{x}}_{2}=A_{22} \hat{x}_{2}+u_{2}+K_{2}\left(y-C_{2} \hat{x}_{2}\right) \\
\dot{P}=A P+P A^{\top}-P C^{\top} Q(t) C P+V(t)
\end{array}\right.
$$

with $P(0)$ a symmetric positive definite matrix, $\mathrm{Q}$ and $\mathrm{V}$ bounded continuous symmetric positive semidefinite matrixvalued functions, and $K:=\left[K_{1}^{\top}, K_{2}^{\top}\right]^{\top}$ given by

$$
K=k(t) P C^{\top} Q, \quad \text { with } 0.5 \leq k(t) \leq k_{\max }<\infty .
$$

Let us recall the definition of uniform observability of the following Linear Time Varying (LTV) system,

$$
\left\{\begin{array}{l}
\dot{x}=\bar{A}(t) x+\bar{B}(t) u \\
y=\bar{C}(t) x
\end{array}\right.
$$

which is instrumental for the stability of the observer in (3).
Given a continuous $n \times n$-dimensional matrix-valued function $\bar{A}(t)$, a continuous $m \times n$-dimensional matrixvalued function $\bar{C}(t)$, and a continuous $m \times m-$ dimensional matrix-valued function $Q(t)>0$ (a positive definite matrix for all $t \in \mathbb{R}^{+}$), the Riccati observability Gramian associated with the triplet $(\bar{A}, \bar{C}, Q)$ is the non-negative definite matrixvalued function defined by

$W_{Q}^{\bar{A}, \bar{C}}(t, t+\delta):=\frac{1}{\delta} \int_{t}^{t+\delta} \Phi^{\top}(s, t) \bar{C}^{\top}(s) Q(s) \bar{C}(s) \Phi(s, t) d s$

where $\Phi\left(t, t_{0}\right)$ is the transition matrix associated with $\bar{A}(t)$, i.e. $\frac{d}{d t} \Phi\left(t, t_{0}\right)=\bar{A}(t) \Phi\left(t, t_{0}\right), \Phi\left(t_{0}, t_{0}\right)=I$.

If $\bar{A}(t)$ and $\bar{C}(t)$ are bounded and if there exists $\delta>0$ and $\epsilon>0$ such that $W_{I_{n}}^{\bar{A}, \bar{C}}(t, t+\delta)>\epsilon I_{n}$ for all $t \geq 0$, then we say that the pair $(\bar{A}, \bar{C})$ is uniformly observable.

Define $\tilde{x}_{2}:=x_{2}-\hat{x}_{2}$, then, from Corollary 3.2 in [12], $\left(\tilde{x}_{1}, \tilde{x}_{2}\right)=(0,0)$ is locally exponentially stable if $Q(t)$ and $V(t)$ are both larger than some positive matrix and the pair $\left(A(0, t), C\left(0, x_{2}, t\right)\right)$ is uniformly observable.

\section{Measurements and System Equations}

Let $\{\mathcal{I}\}$ denotes an inertial (fixed) frame of reference and $\{\mathcal{B}\}$ denotes a frame of reference attached to the camera. Let $R \in \mathrm{SO}(3)$ denote the orientation of the camera frame $\{\mathcal{B}\}$ w.r.t. the inertial frame $\{\mathcal{I}\}$. Let $\bar{\xi}$ and $\bar{v}$ denote the position and the velocity of the camera frame $\{\mathcal{B}\}$ w.r.t. $\{\mathcal{I}\}$ expressed in $\{\mathcal{B}\}$, respectively. Then, the camera second order kinematics equation are

$$
\left\{\begin{array}{l}
\dot{R}=R \omega_{\times} \\
\dot{\bar{\xi}}=-\omega_{\times} \bar{\xi}+\bar{v} \\
\dot{\bar{v}}=-\omega_{\times} \bar{v}+a_{B}+g R^{\top} e_{g}
\end{array}\right.
$$

where $a_{B}$ denotes the specific acceleration expressed in the camera frame $\{\mathcal{B}\}, g$ denotes the gravitational acceleration and the unit vector $e_{g}$ denotes the gravitational acceleration direction expressed in the inertial frame.

The camera is equipped with a strap-down 6DOF IMU composed of a 3-axis gyroscope, which measures the angular velocity $\omega^{m} \in \mathbb{R}^{3}$, and a 3 -axis accelerometer, which measures the specific acceleration $a_{B}^{m} \in \mathbb{R}^{3}$; both measurements are expressed in the camera frame $\{\mathcal{B}\}$. The rate gyros and accelerometer are usually modeled as

$$
\begin{aligned}
\omega^{m} & =\omega-b_{w}-\mu_{w} \\
a_{B}^{m} & =a_{B}-b_{a}-\mu_{a}
\end{aligned}
$$

where $\mu_{w}, \mu_{a}$ denote stochastic additive noises and $b_{w}, b_{a}$ denote constant or slowly time-varying biases.

In this paper we ignore the noise $\left(\mu_{w}=\mu_{a}=0\right)$ and for the sake of simplicity we assume that the gyro bias is zero $\left(b_{w}=0\right)$ or already well calibrated by averaging the gyros measures when the IMU is at rest. The accelerometer calibration is more difficult because it cannot be performed off-line in a resting position and an incorrect calibration can yield drifts in the error (double integration). The following simplified sensor model is considered in this paper

$$
\begin{aligned}
\omega^{m} & =\omega \\
a_{B}^{m} & =a_{B}-b_{a}, \quad \dot{b}_{a}=-\omega_{\times} b_{a} .
\end{aligned}
$$


We assume to have a collection of $n$ source points whose coordinates $\stackrel{\circ}{P}_{i}$ w.r.t. the inertial frame $\{\mathcal{I}\}$ expressed in the basis of $\{\mathcal{I}\}$ are known, and whose calibrated projective coordinates $p_{i}^{p}$ onto the camera plane expressed w.r.t. the camera frame in the basis of $\{\mathcal{B}\}$ are also known, see Fig. 1. Denoting by $P_{i}$ the coordinates of the ith source point w.r.t. the body-frame $\{\mathcal{B}\}$ expressed in $\{\mathcal{B}\}$, and by $z_{i}$ its third component, one verifies $p_{i}^{p}=P_{i} / z_{i}$.

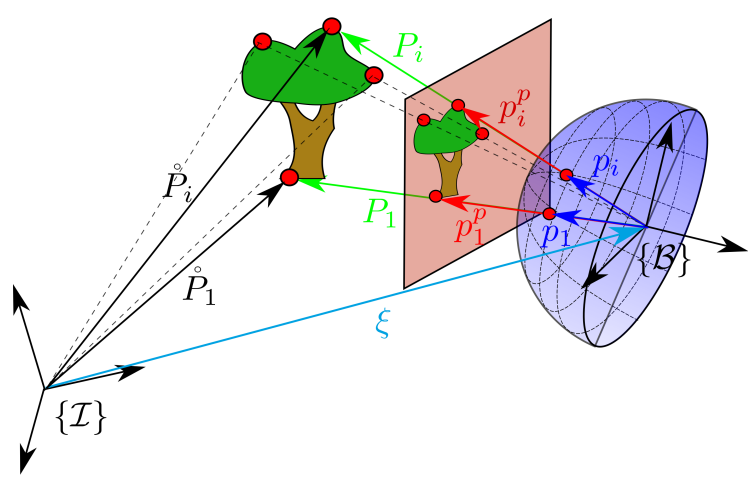

Fig. 1. Intuitive representation of inertial coordinates $\stackrel{\circ}{P}_{i}$, planar projective coordinates $p_{i}^{p}$ and spherical projective coordinates $p_{i}$ of the ith source point.

Rather than using the perspective outputs typically used in computer vision algorithms, we use direction output representation of the measurement $p_{i}:=p_{i}^{p} /\left|p_{i}^{p}\right|$ corresponding to projection onto a virtual spherical image plane and differing from perspective outputs only in the scaling. The unitary vector $p_{i}$ characterizes the bearing (direction) of $P_{i}$ in the basis of $\{\mathcal{B}\}$. Let $\xi$ represent the position of the camera frame with respect to the inertial frame expressed in $\{\mathcal{I}\}$, then one verifies

$$
p_{i}:=\frac{p_{i}^{p}}{\left|p_{i}^{p}\right|}=-R^{\top} \frac{\xi-\stackrel{\circ}{P}_{i}}{\left|\xi-\stackrel{\circ}{P}_{i}\right|} .
$$

\section{Riccati OBSERVER DESIGN}

\section{A. Problem Formulation and observer design}

The problem at hand is to provide an estimate of $\bar{\xi}, \bar{v}$, $b_{a}$ and $R$ given the measurement of the specific acceleration $a_{B}^{m}$, the gyro measurement $\omega^{m}$, and given a set of source points whose positions in the inertial frame $\stackrel{\circ}{P}_{i}$ and bearings $p_{i}$ are known.

To this purpose, we proceed by showing that the system considered can be written in the form (1). In order to do so, as already mentioned in the previous section, we have to define the attitude error between the camera orientation and the estimated one, and then consider a minimal parametrization of the three-dimensional Lie Group $\mathrm{SO}(3)$.

Let $\hat{R}$ be an estimate of $R$ and consider the following pre-observer attitude kinematics

$$
\dot{\hat{R}}=\hat{R} \sigma_{\times}
$$

where $\sigma$ is an innovation term to be designed.
Denoting by $\tilde{R}:=\hat{R}^{\top} R$ the attitude error, one verifies that the error kinematics satisfy

$$
\dot{\tilde{R}}=\tilde{R}\left(\omega-\tilde{R}^{\top} \sigma\right)_{\times},
$$

and by considering the gyros measurement model in (7) one has

$$
\dot{\tilde{R}}=\tilde{R}\left(\omega^{m}-\tilde{R}^{\top} \sigma\right)_{\times} .
$$

The attitude parametrization here chosen is the vector part $\tilde{\tilde{\lambda}} \in \mathcal{B}_{1}^{3}$ of the Rodriguez unit quaternion $\tilde{\Lambda}=\left(\tilde{\lambda}_{0}, \tilde{\lambda}\right)$, where $\tilde{\lambda}_{0} \in \mathcal{B}_{1}^{1}$ denotes the scalar part. The rotation matrix error $\tilde{R}$ is related to $\tilde{\Lambda}$ by the Rodriguez formula

$$
\tilde{R}=I_{3}+2 \tilde{\lambda}_{\times}\left(\tilde{\lambda}_{0} I_{3}+\tilde{\lambda}_{\times}\right) .
$$

Then, in view of (9) and using the fact that $\tilde{\lambda}_{0}=1-|\tilde{\lambda}|^{2}$, one has

$$
\left\{\begin{array}{l}
\tilde{R}=I_{3}+2 \tilde{\lambda}_{\times}+O\left(|\tilde{\lambda}|^{2}\right) \\
2 \dot{\tilde{\lambda}}=\tilde{\omega}-\omega_{\times}^{m} 2 \tilde{\lambda}+O(|\tilde{\lambda}||\tilde{\omega}|)+O\left(|\tilde{\lambda}|^{2}\right)
\end{array}\right.
$$

where $\tilde{\omega}:=\omega^{m}-\sigma$.

Concerning the dynamics of $\bar{v}$ one has

$$
\begin{aligned}
\dot{\bar{v}} & =-\omega_{\times}+g R^{\top} \hat{R} \hat{R}^{\top} e_{g}+a_{B} \\
& =-\omega_{\times}+g \tilde{R}^{\top} \hat{R}^{\top} e_{g}+a_{B} .
\end{aligned}
$$

Substituting $\tilde{R}^{\top}$ with the first order approximation in (10) yields

$$
\dot{\bar{v}}=-\omega_{\times} \bar{v}+g \hat{R}^{\top} e_{g}+a_{B}+g\left(\hat{R}^{\top} e_{g}\right)_{\times} 2 \tilde{\lambda}+O\left(|\tilde{\lambda}|^{2}\right),
$$

and by taking into account the sensor model one obtains

$\dot{\bar{v}}=-\omega_{\times}^{m} \bar{v}+g \hat{R}^{\top} e_{g}+a_{B}^{m}+b_{a}+g\left(\hat{R}^{\top} e_{g}\right)_{\times} 2 \tilde{\lambda}+O\left(|\tilde{\lambda}|^{2}\right)$.

Note that the term $g\left(\hat{R}^{\top} e_{g}\right)_{\times} 2 \tilde{\lambda}$ that appears in the equation above represents a coupling term between the angular and the linear dynamics. This term will appear in the lower diagonal part of the $A\left(\tilde{x}_{1}, t\right)$ matrix in (1), namely $A_{21}\left(\tilde{x}_{1}, t\right)$, making the problem of computing the transition matrix associated with $A\left(\tilde{x}_{1}, t\right)$ very difficult. In order to simplify the subsequent analysis we consider the following change of variable

$$
\bar{b}_{a}:=b_{a}+g\left(\hat{R}^{\top} e_{g}\right)_{\times} 2 \tilde{\lambda} .
$$

Let $\hat{\bar{b}}_{a}$ denotes an estimate of $\bar{b}_{a}$, and denote by $\tilde{\bar{b}}_{a}:=\bar{b}_{a}-\hat{\bar{b}}_{a}$ the new bias estimation error. One verifies that in steady state $\left(\tilde{\lambda}, \tilde{\bar{b}}_{a}\right) \rightarrow(0,0)$ implies $\bar{b}_{a}=b_{a}$, and thus the new change of variable is a valid choice for the accelerometer bias estimation. We proceed, then, by computing the first order approximation of the time derivative of the new bias

$$
\begin{aligned}
\dot{\bar{b}}_{a}= & \frac{d}{d t}\left(b_{a}+g\left(R^{\top} e_{g}\right)_{\times} 2 \tilde{\lambda}+O\left(|\tilde{\lambda}|^{2}\right)\right) \\
= & -\omega_{\times} b_{a}-\omega_{\times} g\left(\hat{R}^{\top} e_{g}\right)_{\times} 2 \tilde{\lambda}+g\left(\hat{R}^{\top} e_{g}\right) \tilde{\omega}+ \\
& +O(|\tilde{\lambda}||\tilde{\omega}|)+O\left(|\tilde{\lambda}|^{2}\right)+\frac{d}{d t} O\left(|\tilde{\lambda}|^{2}\right),
\end{aligned}
$$

one verifies from (10) that $\frac{d}{d t} O\left(|\tilde{\lambda}|^{2}\right)=O(|\tilde{\lambda}||\tilde{\omega}|)+O\left(|\tilde{\lambda}|^{2}\right)$, bearing in mind the expression of $\bar{b}_{a}$ in (12) and by taking into account the sensor model one has

$$
\dot{\bar{b}}_{a}=-\omega_{\times}^{m} \bar{b}_{a}+g\left(\hat{R}^{\top} e_{g}\right)_{\times} \tilde{\omega}+O(|\tilde{\lambda}||\tilde{\omega}|)+O\left(|\tilde{\lambda}|^{2}\right) .
$$


Concerning the output function for the ith source point one has

$$
\begin{aligned}
0 & =-\left|\xi-\stackrel{\circ}{P}_{i}\right| \Pi_{p_{i}} p_{i} \\
& =\Pi_{p_{i}} \bar{\xi}-\Pi_{p_{i}} R^{\top} \stackrel{\circ}{P}_{i} \\
& =\Pi_{p_{i}} \bar{\xi}-\Pi_{p_{i}} \tilde{R}^{\top} \hat{R}^{\top} \stackrel{\circ}{P}_{i} \\
& =\Pi_{p_{i}} \bar{\xi}-\Pi_{p_{i}}\left(I+2 \tilde{\lambda}_{\times}+O\left(|\tilde{\lambda}|^{2}\right)\right)^{\top} \hat{R}^{\top} \stackrel{\circ}{P}_{i} \\
& =\Pi_{p_{i}} \bar{\xi}-\Pi_{p_{i}} R^{\top} \stackrel{\circ}{P}_{i}-\Pi_{p_{i}}\left(R^{\top} \stackrel{\circ}{P}_{i}\right)_{\times} 2 \tilde{\lambda}+O\left(|\tilde{\lambda}|^{2}\right)
\end{aligned}
$$

and thus by setting $y_{i}:=\Pi_{p_{i}} R^{\top} \stackrel{\circ}{P}_{i}$, one obtains

$$
y_{i}=\Pi_{p_{i}} \bar{\xi}-\Pi_{p_{i}}\left(R^{\top} \stackrel{\circ}{P}_{i}\right)_{\times} 2 \tilde{\lambda}+O\left(|\tilde{\lambda}|^{2}\right)
$$

for $i=1, \ldots, n$.

Finally, setting $\tilde{x}_{1}=2 \tilde{\lambda}, x_{2}=\left[\bar{\xi}^{\top}, \bar{v}^{\top}, \bar{b}_{a}^{\top}\right]^{\top}, y=$ $\left[y_{1}^{\top}, \ldots, y_{n}^{\top}\right]^{\top}$ from (6), (10), (11), (13) one obtains the system equation (1) with

$$
\left\{\begin{array}{rlrl}
A_{11}= & -\omega_{\times}^{m}, & A_{21}=0, & u_{1}=\tilde{\omega} \\
A_{22}= & {\left[\begin{array}{ccc}
-\omega_{\times}^{m} & I_{3} & 0_{3} \\
0_{3} & -\omega_{\times}^{m} & I_{3} \\
0_{3} & 0_{3} & -\omega_{\times}^{m}
\end{array}\right],} & u_{2}=\left[\begin{array}{c}
0_{3 \times 1} \\
a_{B}^{m}+g \hat{R}^{\top} e_{g} \\
g\left(\hat{R}^{\top} e_{g}\right)_{\times} \tilde{w}
\end{array}\right] \\
C_{1}=\left[\begin{array}{ccc}
-\Pi_{p_{1}}\left(\hat{R}^{\top} \stackrel{\circ}{P}_{1}\right)_{\times} \\
\vdots \\
-\Pi_{p_{n}}\left(\hat{R}^{\top} \stackrel{\circ}{P}_{n}\right)_{\times}
\end{array}\right], & C_{2}=\left[\begin{array}{ccc}
\Pi_{p_{1}} & 0_{3} & 0_{3} \\
\vdots & \vdots & \vdots \\
\Pi_{p_{n}} & 0_{3} & 0_{3}
\end{array}\right]
\end{array}\right.
$$

Then, the observer is given by (3), (8) with $\sigma=\omega^{m}-u_{1}$.

\section{B. Observability Analysis}

In this section we provide sufficient conditions that ensure good conditioning of the Continuous Riccati Equation (CRE) and the exponential stability of the origin of the observer error as well.

Proposition 1. Consider system (6) with measurement given by (7), and the observer given by (3) along with (8) and (15). Choose $Q(t)$ and $V(t)$ of (3) larger than some positive matrix. Assume that camera's position $\bar{\xi}$, linear velocity $\bar{v}$, angular velocity $\omega$ are bounded for all time. If the pair $\left(A(0, t), C\left(0, x_{2}, t\right)\right)$ is uniformly observable then, the origin of the observer error $\left(\tilde{x}_{1}, \tilde{x}_{2}\right)=(0,0)$ is locally exponentially stable.

Proof. The result is a direct application of Corollary 3.2 in [12].

As shown in [12], the uniform observability of pair $\left(A(0, t), C\left(0, x_{2}, t\right)\right)$ is required to ensure the local uniform exponential stability of the observer error. We show next the condition under which the uniform observability is violated.

Proposition 2. The pair $\left(A(0, t), C\left(0, x_{2}, t\right)\right)$ is not uniformly observable if the following conditions are fulfilled:

- The number of source points is less than or equal to two $(n \leq 2)$.

- All source points are aligned independently of their number $(n \geq 3)$.

- In case of three non-aligned source points $(n=3)$ :
- Static Case: The camera lies on the circular cylinder generated by the circle passing through the three source points and whose axis is orthogonal to the plane containing the source points, known in literature as dangerous cylinder.

- Moving Case: The camera moves along a straight line orthogonal to the plane containing the source points and passing through a source point.

- In case of four and more non-aligned source points $(n \geq 4)$ :

- Static Case: The source points are located on a horopter curve, which is uniquely determined by four points, and the camera frame $\{\mathcal{B}\}$ lies at the origin of this curve.

Proof. In view of (15) one verifies that the transition matrix associated with the state matrix $A$ in (2) is of the form

$$
\Phi(s, t)=\left[\begin{array}{cccc}
\bar{R}_{(s, t)} & 0_{3} & 0_{3} & 0_{3} \\
0_{3} & \bar{R}_{(s, t)} & \bar{R}_{(s, t)}(s-t) & \bar{R}_{(s, t)} \frac{(s-t)^{2}}{2} \\
0_{3} & 0_{3} & \bar{R}_{(s, t)} & \bar{R}_{(s, t)}(s-t) \\
0_{3} & 0_{3} & 0_{3} & \bar{R}_{(s, t)}
\end{array}\right]
$$

where $\bar{R}_{(s, t)}:=\bar{R}^{\top}(s) \bar{R}(t)$.

In view of (5) the pair $(A, C)$ is not uniformly observable if there exists a non-zero constant vector $\nu \in \mathbb{R}^{12}$ such that

$$
C(s) \Phi(s, t) \nu=0,
$$

with $s \in[t, t+\delta]$ and $t$ constant.

Substituting the expression of $C\left(0, x_{2}, s\right)$ in (15) and the one of $\Phi(s, t)$ in (16) on the equation above yields

$$
\Pi_{p_{i}^{\mathcal{I}}}\left[\begin{array}{llll}
-\stackrel{\circ}{P}_{i \times} & I_{3} & I_{3}(s-t) & I_{3} \frac{(s-t)^{2}}{2}
\end{array}\right] \bar{\nu}=0
$$

for $\forall i \in\{1, \ldots, n\}$ and $s \in[t, t+\delta]$; where $p_{i}^{\mathcal{I}}:=-(\xi(t)-$ $\stackrel{\circ}{P}) /|\xi(t)-\stackrel{\circ}{P}|$ denotes the bearing of the ith source point expressed in the inertial frame $\{\mathcal{I}\}$, and $\bar{\nu}=R(t) \nu$.

Let $\bar{\nu}:=\left[\bar{\nu}_{1}^{\top}, \bar{\nu}_{2}^{\top}, \bar{\nu}_{3}^{\top}, \bar{\nu}_{4}^{\top}\right]^{\top}$, with $\bar{\nu}_{1}, \bar{\nu}_{2}, \bar{\nu}_{3}, \bar{\nu}_{4} \in \mathbb{R}^{3}$. Equality (17) characterizes body position trajectories along which the body pose is not uniformly observable. For the case of only one bearing measurement, namely $n=1$, it is trivial to verify that there exists an infinity of solutions for $\bar{\nu}$ such that (17) is satisfied. For instance $\bar{\nu}_{1}=\stackrel{\circ}{P}_{1} /\left|\stackrel{\circ}{P}_{1}\right|$, $\bar{\nu}_{2,3,4}=0$ is a non null solution to (17).

Consider now $n \geq 2$. By choosing a particular value of $s$ equal to $t$ (i.e. $s=t$ ) one deduces from (17) that

$$
\Pi_{p_{i}^{\mathcal{I}}}\left[\begin{array}{ll}
-\stackrel{\circ}{P}_{i \times} & I_{3}
\end{array}\right]\left[\begin{array}{l}
\bar{\nu}_{1} \\
\bar{\nu}_{2}
\end{array}\right]=0
$$

for $\forall i \in\{1, \ldots, n\}$, and subsequently (from (17) and (18))

$$
(s-t) \Pi_{p_{i}^{\mathcal{I}} \bar{\nu}_{3}}+\frac{(s-t)^{2}}{2} \Pi_{p_{i}^{\mathcal{I}} \bar{\nu}_{4}}=0, \quad \forall s \in[t, t+\delta]
$$

This implies that

$$
\left\{\begin{array}{l}
\Pi_{p_{i}^{\mathcal{I}} \bar{\nu}_{3}}=0 \\
\Pi_{p_{i}^{\mathcal{I}} \bar{\nu}_{4}}=0
\end{array}\right.
$$

$\forall i \in\{1, \ldots, n\}$, which in turn implies $\bar{\nu}_{3}=\bar{\nu}_{4}=0$. Then from (18) the proof follows the same arguments as the proof in [12] for the case of mobile velocity measurements. 


\section{EXPERIMENTAL RESULTS}

In this section, we present experimental results to demonstrate the performance of the proposed observer. The experiments were performed on a custom platform composed of a low-cost IMU myAHRS+ and an oCam camera (see Figure 2). The camera mounts a standard M12 lens with $3.6 \mathrm{~mm}$ focal length and $65^{\circ}$ field of view. Images are acquired with a resolution of $640 \times 480$ pixels at 30 frames per second. Inertial data from the IMU are acquired at rates of $100 \mathrm{~Hz}$. The point detection is performed with the ArUco library [20] which is included in OpenCV. Only one fiducial marker (size: $21 \mathrm{~cm}$, dictionary: DICT_4X4_50) has been used during the experiments (Fig. 2).

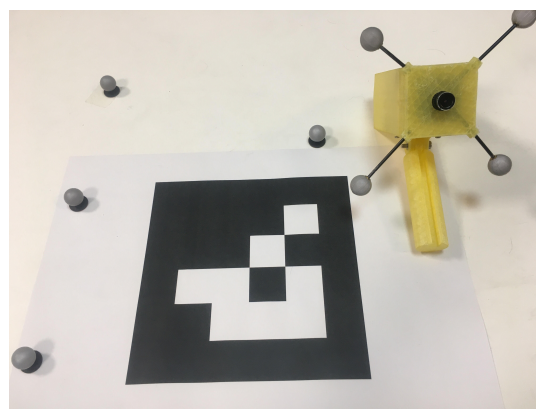

Fig. 2. Experimental platform.

An Optitrack motion capture system (MOCAP) is used together with the markers mounted on the camera and on the fiducial marker in order to provide full pose ground truth measurements. Note that the imperfect positioning of the markers and the initialization process required in Optitrack introduce systematic errors in the ground truth pose.

A discrete version of observer (3) has been implemented on $\mathrm{C}++$ and runs on an HP laptop with Intel ${ }^{\circledR}$ Core $^{\mathrm{TM}} \mathrm{i7}$ $6700 \mathrm{HQ}$ and Nvidia ${ }^{\circledR}$ Quadro M2000M graphic card.

The observer proposed in this paper is compared with the solvePnP() algorithm in OpenCV and with the ground truth data.

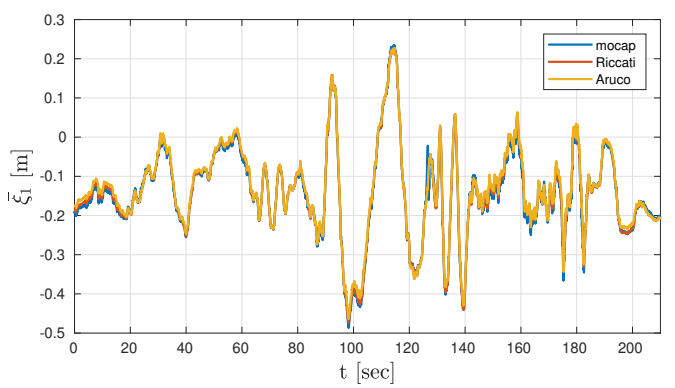

Fig. 3. First component of the camera position $\bar{\xi}$ from MOCAP ground truth, Riccati Observer and ArUco OpenCV algorithm.

Figures 3-4-5 show the time behavior of the estimated position from the Riccati observer (red), ArUco OpenCV (yellow) and the ground truth data (blue). One notes that the position estimates obtained from the Riccati observer and

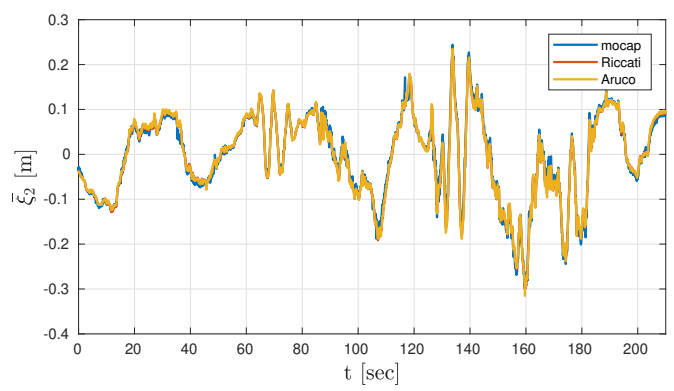

Fig. 4. Second component of the camera position $\bar{\xi}$ from MOCAP ground truth, Riccati Observer and ArUco OpenCV algorithm.

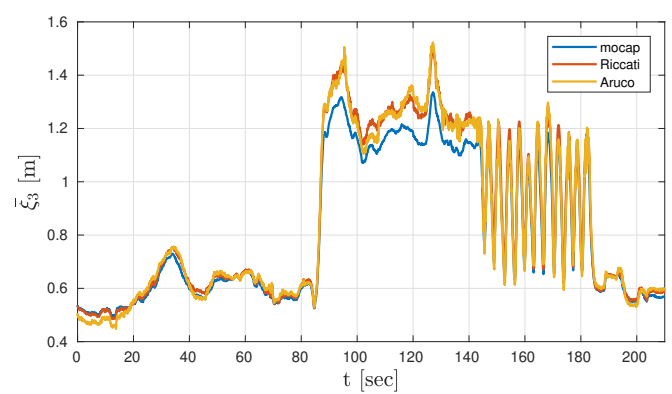

Fig. 5. Third component of the camera position $\bar{\xi}$ from MOCAP ground truth, Riccati Observer and ArUco OpenCV algorithm.

ArUco library are very similar to each other and both are near to the ground truth values except in the third component (Figure 5) when the distance from the target is increased (sec 85-140). The error between the estimated values and the real ones is due to the low resolution of the camera (pixels quantization) along with the ArUco points extraction algorithm.

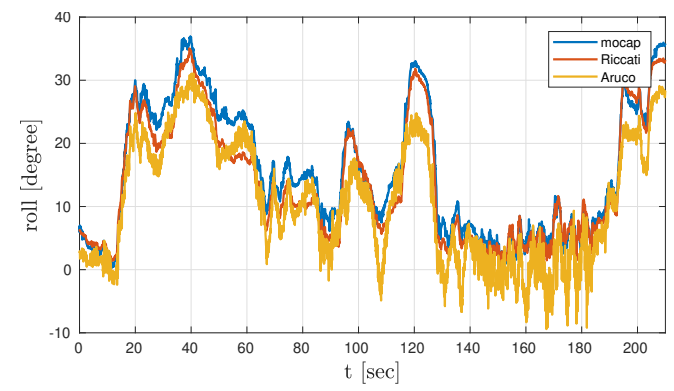

Fig. 6. Roll Euler angle from MOCAP ground truth, Riccati Observer and ArUco OpenCV algorithm.

The time evolutions of the roll, pitch and yaw Euler angles are depicted respectively in Figures 6-7-8 . The proposed observer shows better performances in the attitude estimate with respect to ArUco algebraic method, the difference between the two algorithm is evident when the camera performs complex dynamic motions (sec 145-185). Indeed, during secs 145-185 the camera is moving fast in the third component (Figure 5) and yawing (Figure 8) at the same time; the ArUco algorithm during this time window is switching between two 


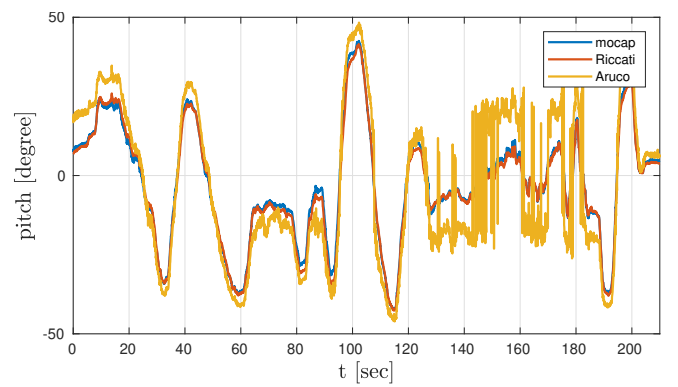

Fig. 7. Pitch Euler angle from MOCAP ground truth, Riccati Observer and ArUco OpenCV algorithm.

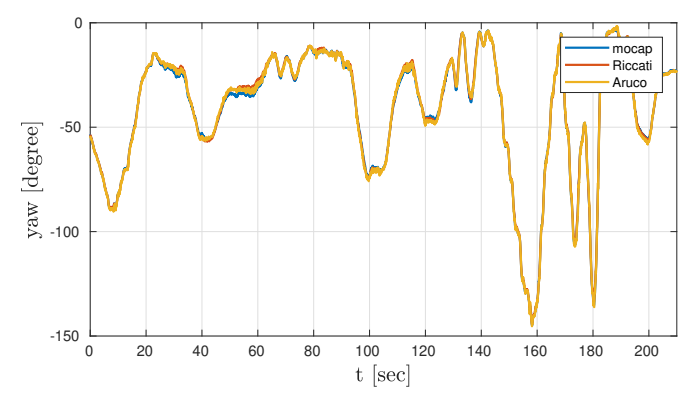

Fig. 8. Yaw Euler angle from MOCAP ground truth, Riccati Observer and ArUco OpenCV algorithm.

possible solutions for the pitch (Figure 7) and roll (Figure 6) Euler angles.

\section{CONCLUSIONS}

In this paper, a novel deterministic observer based on the Riccati framework [12] for the pose and linear velocity estimation of a rigid-body along with accelerometer bias correction has been proposed. The conditions under which the state of the system is not uniformly observable, and thus local exponential stability not guaranteed, have been characterized in terms of the number and position of the source points. Experimental results have been provided as complement to the theoretical approach.

In this work, only one parametrization among the many possible local parametrizations of the pose error has been studied. The parametrization considered is well suited for applications in which the measurements are given in the body-fixed frame.

Future work will focus on different types of measurements that could be available (e.g. barometer, altimeter) along with different pose error parametrizations, and their effects on the accuracy of the observer.

\section{ACKNOWLEDGMENT}

This research was supported by the French DGA-Rapid project "Alcyon", the French EQUIPEX Robotex project and the French Astrid CONGRE project ANR-18-ASTR-0006.

\section{REFERENCES}

[1] N. Gordon A. Doucet, N. de Freitas. An Introduction to Sequential Monte Carlo Methods. Springer, 2001.
[2] N. Aghannan and P. Rouchon. An intrinsic observer for a class of lagrangian systems. IEEE Transactions on Automatic Control, 48(6):936-945, June 2003.

[3] A. P. Aguiar and J. P. Hespanha. Minimum-energy state estimation for systems with perspective outputs and state constraints. In 42nd IEEE International Conference on Decision and Control (IEEE Cat. No.03CH37475), volume 5, pages 4908-4913 Vol.5, Dec 2003.

[4] B.D.O. Anderson and J.B. Moore. Optimal Filtering. Prentice-Hall, Englewood Cliffs, NJ, 1979.

[5] G. Baldwin, R. Mahony, and J. Trumpf. A nonlinear observer for 6 dof pose estimation from inertial and bearing measurements. In 2009 IEEE International Conference on Robotics and Automation, pages 2237-2242, May 2009.

[6] A. Barrau and S. Bonnabel. The invariant extended kalman filter as a stable observer. IEEE Transactions on Automatic Control, 62(4):17971812, April 2017.

[7] S. Berkane and A. Tayebi. Position, velocity, attitude and gyro-bias estimation from imu and position information. In 2019 18th European Control Conference (ECC), pages 4028-4033, June 2019.

[8] S. Bonnabel, P. Martin, and P. Rouchon. Non-linear symmetrypreserving observers on lie groups. IEEE Transactions on Automatic Control, 54(7):1709-1713, July 2009.

[9] Michel Dhome, Marc Richetin, Jean-Thierry Lapresté, and Gérard Rives. The inverse perspective problem from a single view for polyhedra location. Proceedings CVPR '88: The Computer Society Conference on Computer Vision and Pattern Recognition, pages 6166, 1988.

[10] J. Farrell. Aided Navigation: GPS with High Rate Sensors. McGrawHill, Inc., New York, NY, USA, 1 edition, 2008.

[11] J. Grunert. Das pothenotische problem in erweiterter gestalt nebst über seine anwendungen in der geodäsie. Grunerts Archiv für Mathematik und Physiks, (Band 1):238-248, 1841.

[12] T. Hamel and C. Samson. Riccati observers for the nonstationary pnp problem. IEEE Transactions on Automatic Control, 63(3):726-741, 2017.

[13] R. M. Haralick, H. Joo, C. Lee, X. Zhuang, V. G. Vaidya, and M. B. Kim. Pose estimation from corresponding point data. IEEE Transactions on Systems, Man, and Cybernetics, 19(6):1426-1446, Nov 1989.

[14] A. Huster, E. W. Frew, and S. M. Rock. Relative position estimation for auvs by fusing bearing and inertial rate sensor measurements. In Proceedings of the 12th International Symposium on Unmanned Untethered Submersible Thecnology, 2001.

[15] M. Izadi and A. K. Sanyal. Rigid body pose estimation based on the lagrange-d'alembert principle. Automatica, 71:78 - 88, 2016.

[16] S. J. Julier and J. K. Uhlmann. New extension of the Kalman filter to nonlinear systems. In Signal Processing, Sensor Fusion, and Target Recognition VI, volume 3068, pages 182 - 193. International Society for Optics and Photonics, SPIE, 1997.

[17] C. . Lu, G. D. Hager, and E. Mjolsness. Fast and globally convergent pose estimation from video images. IEEE Transactions on Pattern Analysis and Machine Intelligence, 22(6):610-622, June 2000

[18] R. Mahony, T. Hamel, and J. Pflimlin. Nonlinear complementary filters on the special orthogonal group. IEEE Transactions on Automatic Control, 53(5):1203-1218, June 2008.

[19] R. Mahony, J. Trumpf, and T. Hamel. Observers for kinematic systems with symmetry. IFAC Proceedings Volumes, 46(23):617 - 633, 2013. 9th IFAC Symposium on Nonlinear Control Systems.

[20] Francisco J. Romero Ramírez, Rafael Muñoz-Salinas, and Rafael Medina Carnicer. Speeded up detection of squared fiducial markers. Image Vision Comput., 76:38-47, 2018.

[21] J.F. Vasconcelos, R. Cunha, C. Silvestre, and P. Oliveira. A landmark based nonlinear observer for attitude and position estimation with bias compensation. IFAC Proceedings Volumes, 41(2):3446 - 3451, 2008. 17th IFAC World Congress.

[22] O. J. Woodman. An introduction to inertial navigation. Technical Report, University of Cambridge, Computer Laboratory, (UCAM CLTR-696):1-37, 2007. 